\title{
microRNA-450a targets DNA methyltransferase 3a in hepatocellular carcinoma
}

\author{
ZHIHONG WENG $^{1 *}$, DONGDONG WANG ${ }^{2 *}$, WENYUE ZHAO ${ }^{2}$, MENGQI SONG ${ }^{2}$,

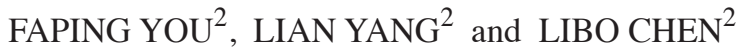 \\ ${ }^{1}$ Department of Hepatology and Infectious Disease; ${ }^{2}$ Hepatobiliary Surgery Center, Union Hospital, \\ Tongji Medical College, Huazhong University of Science and Technology, Wuhan 430022, P.R. China
}

Received April 27, 2011; Accepted June 7, 2011

DOI: $10.3892 /$ etm.2011.288

\begin{abstract}
RNAs (miRNAs) have been proven to play key regulatory roles in hepatocarcinogenesis. In the present study, the possible role of microRNA-450a (miR-450a) in hepatocarcinogenesis was investigated. Our study revealed that miR-450a was significantly down-regulated in hepatocellular carcinoma (HCC) tissues compared with that in normal liver (NL) and para-tumorous (PT) tissues, and miR-450a expression in HepG2 cells was significantly lower than that in L02 cells. Both the mRNA and protein levels of the miR-450a potential target gene, DNA methyltransferase 3a (DNMT3a), were obviously higher in HCC compared with levels in the NL and PT tissues. We further identified DNMT3a as the direct target gene for miR-450a, and ectopic miR-450a expression in HepG2 cells caused the down-regulation of DNMT3a and an inhibition of cell proliferation. Taken together, these findings suggest that miR-450a plays an important regulatory role in hepatocarcinogenesis through inhibition of DNMT3a expression, and miR-450a may be a potential target for the treatment of HCC.
\end{abstract}

\section{Introduction}

Hepatocellular carcinoma (HCC) is one of the most common malignant tumors worldwide, especially in East Asia, including China. However, the pathogenesis of hepatocarcinogenesis is far from clear. microRNAs (miRNAs) are a type of highly conserved non-coding small RNAs which regulate gene

Correspondence to: Dr Libo Chen, Hepatobiliary Surgery Center, Union Hospital, Tongji Medical College, Huazhong University of Science and Technology, Wuhan 430022, P.R. China

E-mail: libo_chen@hotmail.com

*Contributed equally

Abbreviations: HCC, hepatocellular carcinoma; DNMT3a, DNA methyltransferase 3a; miRNA, microRNA; UTR, untranslated region

Key words: hepatocellular carcinoma, microRNA-450a, DNA methyltransferase $3 \mathrm{a}$ expression at the post-transcriptional level. It is now clear that miRNAs can potentially regulate every aspect of cellular activity, including differentiation and development, metabolism, proliferation, and apoptotic and viral infection. Recently, miRNAs have been found to play pivotal roles in many malignancies including HCC development (1-9). The presence of a molecular prognostic miRNA signature in primary HCC clinical specimens has also been confirmed by several recent studies (4,6,7,10-12), and many miRNAs have been found to play important regulatory roles in hepatocarcinogenesis.

Our previous study found that miRNA-602 has an important regulatory activity in $\mathrm{HBV}$-mediated hepatocarcinogenesis by inhibiting the tumor-suppressive gene RASSF1A from very early stages of chronic HBV hepatitis to HBV-positive cirrhosis to HCC (13). In this study, the expression of miRNA-450a and its potential target, DNA methyltransferase 3a (DNMT3a), was investigated in $\mathrm{HCC}$.

\section{Materials and methods}

Patients and cell lines. Histologically normal liver samples were obtained by biopsy during surgery from eight patients with gallbladder stones. Thirty-four HCC and corresponding non-malignant para-tumorous specimens were collected by radical hepatectomy. All tissues were obtained with informed consent from the patients, and were verified by biochemistry and pathological examination. The study was approved by the Institutional Review Board of Tongji Medical College, Huazhong University of Science and Technology (China). The cell lines, HepG2 and L02, were cultured in RMPI-1640 with $10 \%$ fetal bovine serum.

microRNA arrays. miR-450a from 8 normal livers, $34 \mathrm{HCC}$, and corresponding non-tumorous tissues was analyzed. microRNA arrays were performed as described previously (13). Briefly, 100 ng RNA of each specimen was extracted using TRIzol (Invitrogen) and an RNeasy Mini kit (Qiagen) according to the manufacturer's instructions. The samples were hybridized on a hybridization station. Scanning was performed with an Axon GenePix 4000B microarray scanner.

Quantitative real-time PCR. Total-RNA was extracted from the tissues and cell lines by TRIzol reagent (Invitrogen), according 
to the manufacturer's instructions. For miRNA qPCR, reverse transcription was performed using the QuantiMir RT kit (System Biosciences). Primers for miR-450a were forward, 5'-TTTTGCGATGTGTTCC-3' and reverse, 5'-GTGCAG GGTCCGAGGT-3'; and for control U6 forward, 5'-CTCGCT TCGGCAGCACA-3' and reverse, 5'-AACGCTTCACGAATT TGCGT-3'. Primers for DNMT3a were forward, 5'-CAATGA CCTCTCCATCGTCAAC-3' and reverse, 5'-CATGCAGGA GGCGGTAGAA-3'; and for $\beta$-actin forward, 5'-GAACGG TGAAGGTGACAG-3' and reverse, 5'-TAGAGAGAAGTG GGGTGG-3'.

The amplification of miR-450a was performed as follows: denaturation at $95^{\circ} \mathrm{C}$ for $10 \mathrm{~min}$, followed by 40 cycles of $95^{\circ} \mathrm{C}$ for $10 \mathrm{sec}, 60^{\circ} \mathrm{C}$ for $20 \mathrm{sec}$, and $72^{\circ} \mathrm{C}$ for $10 \mathrm{sec}$. Amplification of DNMT3a was performed as follows: denaturation at $95^{\circ} \mathrm{C}$ for $10 \mathrm{~min}$, followed by 40 cycles of $95^{\circ} \mathrm{C}$ for $15 \mathrm{sec}$ and $60^{\circ} \mathrm{C}$ for $1 \mathrm{~min}$. U6 RNA was used as an miRNA internal control, and $\beta$-actin was used to normalize the amount of total-mRNA in each sample. All values were calculated as ratios normalized to U6 or $\beta$-actin.

Transfection of miR-450a mimics into HepG2 cells. Synthesized miR-450a mimics were purchased from Dharmacon (Lafayette, CA). HepG2 cells were cultured in RPMI-1640 plus $10 \%$ fetal bovine serum. After reaching 30 to $50 \%$ confluency, the cells were transfected with $60 \mathrm{nM}$ of the miR-450a mimics or an miRNA mimic control. Cell proteins were harvested and measured $72 \mathrm{~h}$ after transfection.

Western blot analyses. Western blotting was performed as described previously (13). Briefly, cell lysates were electrophoresed on 10 to $20 \%$ polyacrylamide gels (Bio-Rad) and transferred to Immobilon-PSQ membranes (Millipore, MA). The membranes were blocked with TBS containing 5\% skim milk and $0.1 \%$ Tween-20, and then incubated with a primary antibody. Anti-DNMT3a and anti-GAPDH antibodies (CST for DNMT3a and ProMab for GAPDH) were used according to the manufacturer's instructions. GAPDH was used as an internal control, and all values were calculated as ratios normalized to GAPDH.

Luciferase activity assay. The 3'UTR of DNMT3a containing an intact miR-450a recognition sequence was amplified by PCR and inserted into a pGL3 vector (Promega) immediately downstream of the luciferase gene. A pGL3 construct containing DNMT3a 3'UTR with point mutations in seed sequence was synthesized using a site-directed mutagenesis kit (Stratagene, USA) according to the manufacturer's instructions. The primer for DNMT3a was forward, 5'-GCTCTAGACGAAAAGGGTTGGACATCAT-3' and reverse, 5'-GCTCTAGAGCCGAGGGAGTCTCCTTTTA-3'.

Cells $\left(2 \times 10^{5}\right)$ were co-transfected with $500 \mathrm{ng}$ of the pGL3-DNMT3a-WT or pGL3-DNMT3a-MUT constructs with miR-450a mimics or a negative control. Each sample was co-transfected with $50 \mathrm{ng}$ of pRL-TK plasmid expressing Renilla luciferase to monitor the transfection efficiency (Promega). A luciferase activity assay was performed $48 \mathrm{~h}$ after transfection with the dual luciferase reporter assay system (Promega). The relative luciferase activity was normalized with Renilla luciferase activity.
A

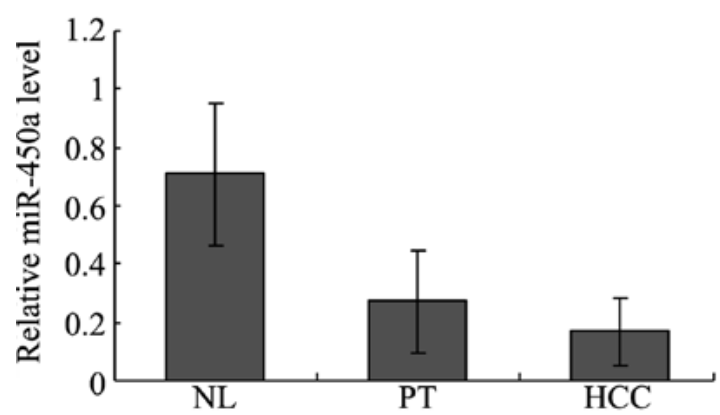

B

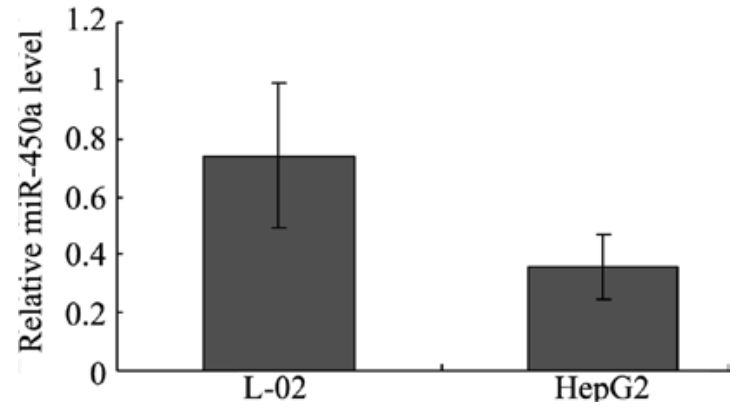

Figure 1. miR-450a expression in HCC tissues and hepatoma cell lines. (A) The relative expression of miR-450a in the normal liver (NL) and corresponding non-cancerous para-tumorous (PT) tissue, and hepatocellular carcinoma (HCC) from $34 \mathrm{HBV}$-positive HCC patients using real-time PCR. (B) The relative expression of miR-450a in the L02 and HepG2 cell lines was also detected using real-time PCR.

Cell apoptosis and proliferation assays. Apoptosis was detected by Annexin V-FITC/PI (KeyGen, China) doublestaining. Briefly, $72 \mathrm{~h}$ after transfection, cells $\left(2 \times 10^{6} / \mathrm{ml}\right)$ were harvested and stained with anti-Annexin $\mathrm{V}$ conjugated with FITC and propidium iodide (PI) for $15 \mathrm{~min}$, and then detection was carried out using FACScan. Data were analyzed using CellQuest software.

Cell proliferation was measured using the Cell Counting Kit-8 (CCK-8) (Beyotime, China). Cells at 0, 10, 20, 30, 40, 50, 60 and $70 \mathrm{~h}$ after transfection were treated with $10 \mu \mathrm{l} \mathrm{CCK}-8$ per well according to the manufacturer's instructions. The absorbance in each well was measured at $450 \mathrm{~nm}$ by a microplate reader.

Statistics. All experiments were repeated 3 times, and data were recorded as mean \pm SD and analyzed using the Student's t-test and one-way ANOVA by SPSS 13.0 software. $\mathrm{P}<0.05$ was considered to be significantly significant.

\section{Results}

Down-regulation of miR-450a is validated in HCC tissues and cell lines. To validate miR-450a expression in the HCC tissues, miR-450a levels were quantified using real-time PCR. Our results showed that the expression of miR-450a was significantly down-regulated in the HCC tissues compared with that in the normal liver (NL) and para-tumorous (PT) tissues. In NL, PT and HCC tissues, the levels were $0.710 \pm 0.245$, $0.271 \pm 0.175,0.168 \pm 0.114$, respectively $(\mathrm{P}<0.05$ for NL vs. PT and NL vs. HCC) (Fig. 1A). miR-450a levels were not significantly different between the PT and HCC groups $(\mathrm{P}>0.05$, 
A

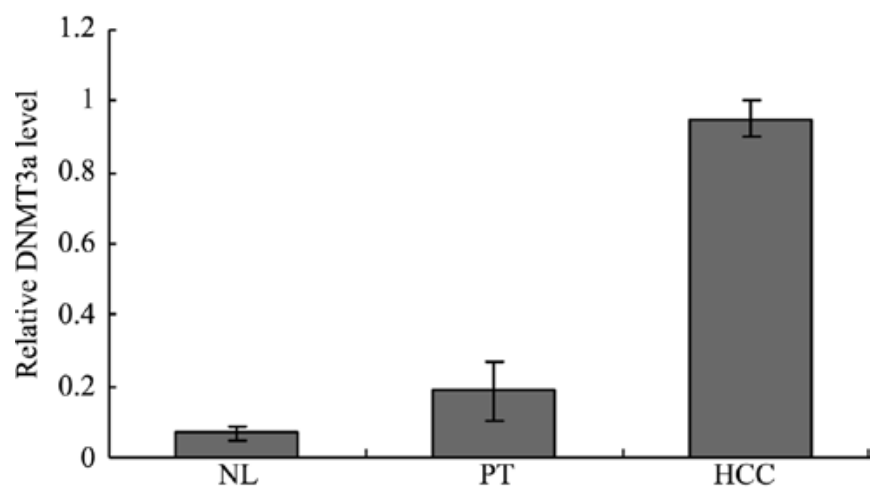

B

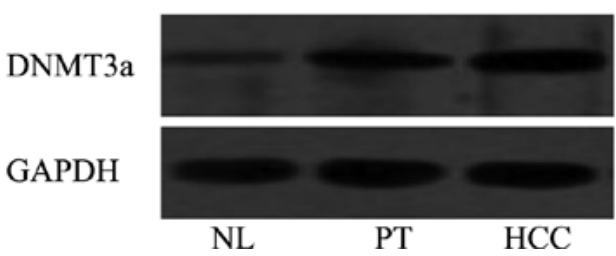

Figure 2. Expression of the miR-450a target gene, DNA methyltransferase 3a (DNMT3a), in various types of liver tissues assayed using real-time PCR and Western blot analysis. (A) mRNA levels of DNMT3a in normal liver (NL), corresponding non-cancerous para-tumorous (PT) tissue and hepatocellular carcinoma (HCC). (B) Protein levels of DNMT3a in NL, PT and HCC.

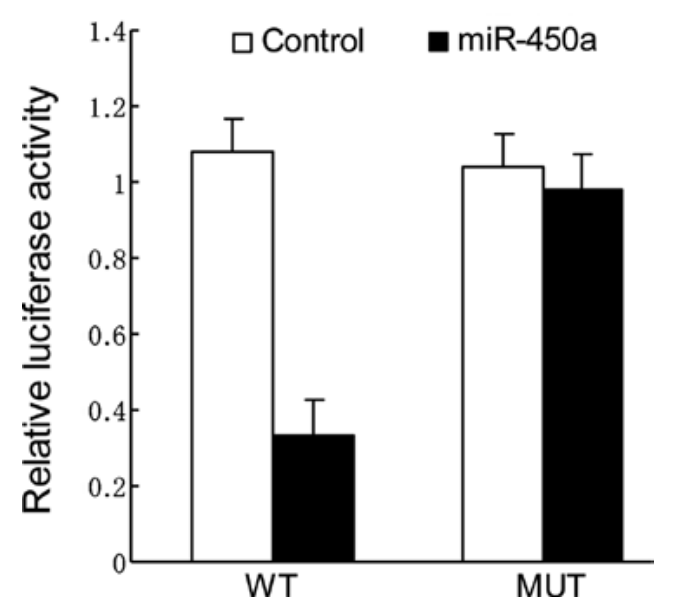

Figure 3. Ectopic miR-450a expression inhibits wild-type (WT) but not mutant (MUT) DNMT3a 3'UTR reporter activity in HepG2 cells. Cells were co-transfected with miR-450a mimics and with either WT or MUT DNMT3a 3'UTR reporter construct. Luciferase activity assay was performed at $48 \mathrm{~h}$ after transfection (Mann-Whitney test, $\mathrm{P}<0.05$ ).

Fig. 1A). The levels of miR-450a in the L02 and HepG2 cells were also evaluated using real-time PCR. The miR-450a level was significantly lower in the HepG2 than that in the L02 cells. In the L02 and HepG2 cells, the levels were $0.740 \pm 0.251$ and $0.358 \pm 0.112$, respectively $(\mathrm{P}<0.01$, Fig. $1 \mathrm{~B})$, which indicated that miR-450a levels may be associated with hepatocellular carcinogenesis.

In silico prediction of an miR-450a target. Bioinformatics analysis suggested that the key enzyme in DNA methylation, DNMT3a, was one of the potential targets of miR-450a. (http://
A

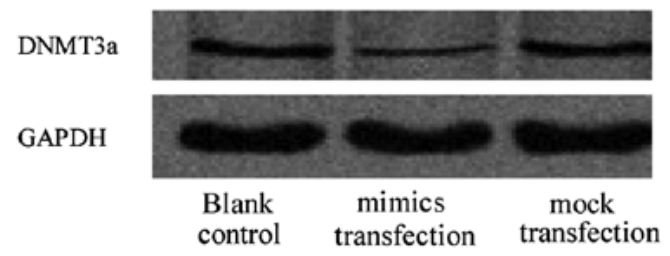

B

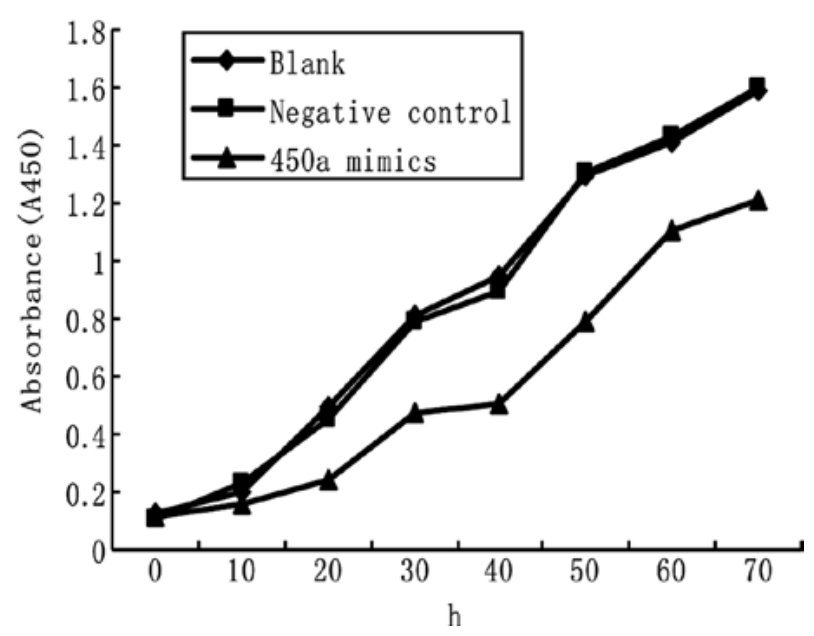

Figure 4. Effects of ectopic miR-450a on DNMT3a expression and hepatoma cell proliferation. (A) miR-450a mimics were transfected into HepG2 cells, and DNMT3a protein levels were measured by Western blot analysis. (B) Cell proliferation was measured by the Cell Counting Kit-8 (CCK-8). Cells at $0,10,20,30,40,50,60$ and $70 \mathrm{~h}$ after transfection were treated with $10 \mu \mathrm{l}$ CCK-8 per well according to the manufacturer's instructions. The absorbance in each well was measured at $450 \mathrm{~nm}$ by a microplate reader.

www.ebi.ac.uk/enright-srv/microcosm/cgi-bin/targets/v5/hit_list. pl?genome_id=\&mirna_id=hsa-mir-450a\&external_name= DNMT3a\&gene_id $=\&$ go_class $=$ function $\&$ go_term $=\&$ logic $=$ phrase\&terms $=$ ). The predicted binding of miR-450a with DNMT3a 3'UTR is illustrated below:

\section{hsa-miR-450a $\quad 3^{\prime} \quad$ U AUAAUCCUUG UGUAG CG UUUU | || || || || | || | | DNMT3a 3'UTR $\quad 5^{\prime}$...A T AG TAACAA T ACC TT GC AGAG...}

Expression of the potential miR-450a target gene DNMT3a is higher in HCC compared with normal liver. Expression of the miR-450a potential target gene DNMT3a in various liver tissues was investigated. The DNMT3a mRNA level in HCC $(0.950 \pm 0.053)$ was significantly higher than levels in the NL $(0.068 \pm 0.017)$ or PT $(0.186 \pm 0.082)$ tissues $(\mathrm{P}<0.05$ for HCC vs NL and HCC vs. PT) (Fig. 2A). The DNMT3a protein level in HCC $(0.522 \pm 0.014)$ was significantly higher than levels in the NL $(0.214 \pm 0.097)$ and PT $(0.400 \pm 0.018)$ tissues $(\mathrm{P}<0.05$ for $\mathrm{HCC}$ vs. NL and $\mathrm{HCC}$ vs. PT) (Fig. 2B).

DNMT3a is the direct target of miR-450a. To validate the miRNA-target interactions, the DNMT3a complementary sites, with or without mutations, were cloned into the 3'UTR of the firefly luciferase gene and co-transfected with miR-450a mimics or a negative control in HepG2 cells. The relative luciferase activity of the wild-type (WT) construct of DNMT3a 
3'UTR in HepG2 cells was significantly reduced in the presence of miR-450a whereas such a suppressive effect was not observed in the cells with the mutant (MUT) construct of DNMT3a 3'UTR (Fig. 3).

Ectopic miR-450a expression down-regulates DNMT3a and inhibits HepG2 cell proliferation. miRNA-450a mimics were transfected into HepG2 cells, and protein levels of the target gene, DNMT3a, were measured. DNMT3a protein level was much lower in the mimic group than those in the blank control or mock group. Levels in the blank control, mimic and mock groups were $0.347 \pm 0.053,0.114 \pm 0.011$ and $0.335 \pm 0.033$, respectively $(\mathrm{P}<0.05$ for mimic vs. blank control and mock) (Fig. 4A).

In order to clarify whether miR-450a deregulation plays a role in hepatocarcinogenesis, the proliferation and apoptosis rates of HepG2 cells were measured after miR-450a exposure. An MTT assay indicated that at 20,30 and $40 \mathrm{~h}$ after miR-450a mimic exposure, the proliferation rate of HepG2 was inhibited to 52,42 and $46.3 \%$ of the control, respectively (Fig. 4B). The differences between the intervention and control groups at 20, 30 , and $40 \mathrm{~h}$ were significant ( $\mathrm{P}<0.05$ for all points) (Fig. 4B), while the difference between the blank and control groups was not significant ( $\mathrm{P}>0.05$; Fig. 4B).

The apoptosis rate of HepG $272 \mathrm{~h}$ after transfection with the miR-450a mimics was not significantly different from that of the HepG2 cells without miR-450a exposure (data not shown).

\section{Discussion}

Maintenance of genomic stability is regulated by both genetic and epigenetic mechanisms. It is well known that promoter hypermethylation mediated by DNA methyltransferases (DNMTs) is the main reason for epigenetic inactivation of tumor-suppressor genes (TSGs). Increasing evidence has revealed that viral genes are important in regulating DNA methylation (14). The epigenetic mechanisms involved in virusassociated cancers are poorly understood. Hypermethylation is responsible for the silencing of TSGs involved in hepatocarcinogenesis. Several recent studies have suggested that HBx is involved in epigenetic regulation during hepatocarcinogenesis $(15,16)$. Previous studies support a role for miRNAs as both targets and effectors in aberrant mechanisms of DNA hypermethylation $(17,18)$. miRNAs are non-coding RNAs, 19-25 nucleotides in length, that regulate gene expression by inducing translational inhibition or cleavage of their target mRNAs through base pairing at partially or fully complementary sites (19). Research groups have shown that miRNAs are altered in human malignancies and can function as tumor-suppressor genes or oncogenes through regulation of expression of their target genes (19). Several studies have shown that specific miRNAs are aberrantly expressed in malignant HCC cells or tissues compared to non-malignant hepatocytes or tissue $(4,11,20-23)$.

In hepatocarcinogenesis proceeding from normal liver to chronic hepatitis/cirrhosis to HCC, increased expression of DNMT mRNA is correlated with a progressive increase in the number of methylated genes (24). Presently three catalytically active DNMTs, namely DNMT1, DNMT3a and DNMT3b, have been identified. Emerging evidence has revealed that levels of DNMT1, DNMT3a and DNMT3b mRNA are reportedly increased in various malignancies, including colorectal, liver, and gastric cancers $(25,26)$. DNMTs and demethyltransferase were found to cooperate with each other, leading to genetic instability that eventually promotes cancer progression (27). Recently, one study demonstrated that a high level of DNMT3a protein was significantly associated with a lower overall survival in lung cancer (18). It has been demonstrated that DNMT3a mediates tumor promotion through its interaction with p53 and the resultant suppression of p53-mediated transcription of tumor-suppressor genes (28).

In the present study, we characterized the role of miR$450 \mathrm{a}$ in the regulation of DNA methylation in HCC for the first time. The results revealed that miR-450a expression was down-regulated to a greater extent in HCC tissues than in corresponding non-cancerous liver tissues, and miR-450a expression in HepG2 cells was significantly lower than that in L02 cells. Additionally our findings indicate that there is a vital link between miR-450a and DNMT3a. First, bioinformatics analysis suggests that the key enzyme in DNA methylation, DNMT3a, is one of the potential targets of miR-450a. Second, our findings indicate that miR-450a expression is inversely correlated with DNMT3a expression in HCC. Down-regulation of miR-450a resulted in an up-regulation of DNMT3a. More importantly, we also provide evidence from the luciferase activity assay that DNMT3a is a direct target of miR-450a. Taken together, our findings confirm that miR-450a regulates DNMT3a expression and may have a tumor-suppressive role in HCC development.

We also provide insights concerning the biological function of miR-450a in HepG2 cells. DNMT3a protein expression was decreased significantly more by restoring miR-450a expression in HepG2 cells transfected with the miR-450a mimics than in the control group. The CCK-8 assay and flow cytometric analysis were also performed in the HepG2 cells. Ectopic miR-450a expression in HepG2 cells caused an inhibition of cell proliferation. These results indicate that the enhanced expression of miR-450a by gene transfer may reverse the malignant phenotypes of HCC cell lines by inhibiting DNMT3a expression.

As the number of samples used for investigating miR-450a down-regulation and the correlation between DNMT3a and miR-450a expression was small, further validation in large cohorts and in independent studies are necessary. Additional studies are needed to investigate the regulatory mechanism of miR-450a expression in order to better understand the mechanism by which miR-450a is down-regulated in HCC. In conclusion, the results from the current study suggest that miR-450a plays an important regulatory role in hepatocarcinogenesis through inhibition of DNMT3a expression, and miR-450a may be a potential target for the treatment of HCC.

\section{Acknowledgements}

The study was supported by the National Natural Science Foundation grants of China (NSFC nos. 30672067, 30700190, 30900663) and a grant from the New-Century Excellent Talent Program of the Education Ministry of China (no. NCET-07318). We also thank Medjaden Bioscience Limited for assisting in the preparation of this manuscript. 


\section{References}

1. Calin GA and Croce CM: MicroRNA signatures in human cancers. Nat Rev Cancer 6: 857-866, 2006.

2. Gregory RI and Shiekhattar R: MicroRNA biogenesis and cancer. Cancer Res 65: 3509-3512, 2005.

3. Lu J, Getz G, Miska EA, et al: MicroRNA expression profiles classify human cancers. Nature 435: 834-838, 2005.

4. Meng F, Henson R, Wehbe-Janek H, Ghoshal K, Jacob ST and Patel T: MicroRNA-21 regulates expression of the PTEN tumor suppressor gene in human hepatocellular cancer. Gastroenterology 133: 647-658, 2007.

5. Gramantieri L, Ferracin M, Fornari F, et al: Cyclin G1 is a target of miR-122a, a microRNA frequently downregulated in human hepatocellular carcinoma. Cancer Res 67: 6092-6099, 2007.

6. Murakami Y, Yasuda T, Saigo K, et al: Comprehensive analysis of microRNA expression patterns in hepatocellular carcinoma and non-tumorous tissues. Oncogene 25: 2537-2745, 2006.

7. Budhu A, Jia HL, Forgues M, et al: Identification of metastasisrelated microRNAs in hepatocellular carcinoma. Hepatology 47: 897-907, 2008

8. Shah YM, Morimura K, Yang Q, Tanabe T, Takagi M and Gonzalez FJ: Peroxisome proliferator-activated receptor alpha regulates a microRNA-mediated signaling cascade responsible for hepatocellular proliferation. Mol Cell Biol 27: 4238-4247, 2007.

9. Huang YS, Dai Y, Yu XF, et al: Microarray analysis of microRNA expression in hepatocellular carcinoma and non-tumorous tissues without viral hepatitis. J Gastroenterol Hepatol 23: 87-94, 2008.

10. Jiang J, Gusev Y, Aderca I, et al: Association of microRNA expression in hepatocellular carcinomas with hepatitis, cirrhosis, and patient survival. Clin Cancer Res 14: 419-427, 2008.

11. Varnholt H, Drebber U, Schulze F, et al: MicroRNA gene expression profile of hepatitis $\mathrm{C}$ virus-associated hepatocellular carcinoma. Hepatology 47: 1223-1232, 2008.

12. Ladeiro Y, Couchy G, Balabaud C, et al: MicroRNA profiling in hepatocellular tumors is associated with clinical features and oncogene/tumor-suppressor gene mutations. Hepatology 47 1955-1963, 2008.

13. Yang L, Ma Z, Wang D, Zhao W, Chen L and Wang G: MicroRNA-602 regulating tumor suppressive gene RASSF1A is overexpressed in hepatitis B virus-infected liver and hepatocellular carcinoma. Cancer Biol Ther 9: 1-6, 2010.

14. Li HP, Leu YW and Chang YS: Epigenetic changes in virusassociated human cancers. Cell Res 15: 262-271, 2005.

15. Park IY, Sohn BH, Yu E, et al: Aberrant epigenetic modifications in hepatocarcinogenesis induced by hepatitis B virus X protein. Gastroenterology 132: 1476-1494, 2007.
16. Tong A, Gou L, Lau QC, et al: Proteomic profiling identifies aberrant epigenetic modifications induced by hepatitis B virus $\mathrm{X}$ protein. J Proteome Res 8: 1037-1046, 2009.

17. Saito Y, Liang G, Egger G, et al: Specific activation of microRNA-127 with downregulation of the proto-oncogene BCL6 by chromatin-modifying drugs in human cancer cells. Cancer Cell 9: 435-443, 2006

18. Fabbri M, Garzon R, Cimmino A, et al: MicroRNA-29 family reverts aberrant methylation in lung cancer by targeting DNA methyltransferases 3A and 3B. Proc Natl Acad Sci USA 104: 15805-15810, 2007.

19. Garzon R, Fabbri M, Cimmino A, Calin GA and Croce C: MicroRNA expression and function in cancer. Trends Mol Med 12: 580-587, 2006.

20. Kutay H, Bai S, Datta J, et al: Downregulation of miR-122 in the rodent and human hepatocellular carcinomas. J Cell Biochem 99: 671-678, 2006.

21. Jacob JR, Sterczer A, Toshkov IA, et al: Integration of woodchuck hepatitis and $\mathrm{N}$-myc rearrangement determine size and histologic grade of hepatic tumors. Hepatology 39: 1008-1016, 2004.

22. Harada H, Nagai H, Ezura Y, et al: Down-regulation of a novel gene, DRLM, in human liver malignancy from $4 \mathrm{q} 22$ that encodes a NAP-like protein. Gene 296: 171-177, 2002.

23. Wong QW, Lung RW, Law PT, et al: MicroRNA-223 is commonly repressed in hepatocellular carcinoma and potentiates expression of Stathmin1. Gastroenterology 135: 257-269, 2008.

24. Oh BK, Kim H, Park HJ, et al: DNA methyltransferase expression and DNA methylation in human hepatocellular carcinoma and their clinicopathological correlation. Int J Mol Med 20: 65-73, 2007.

25. Eads CA, Danenberg KD, Kawakami K, Saltz LB, Danenberg PV and Laird PW: CpG island hypermethylation in human colorectal tumors is not associated with DNA methyltransferase overexpression. Cancer Res 59: 2302-2306, 1999.

26. Ding WJ, Fang JY, Chen XY and Peng YS: The expression and clinical significance of DNA methyltransferase proteins in human gastric cancer. Dig Dis Sci 53: 2083-2089, 2008.

27. Fang JY, Cheng ZH, Chen YX, et al: Expression of DNMT1, demethylase, MeCP2 and methylation of tumor-related genes in human gastric cancer. World J Gastroenterol 10: 3394-3398, 2004.

28. Wang YA, Kamarova Y, Shen KC, et al: DNA methyltransferase$3 \mathrm{a}$ interacts with $\mathrm{p} 53$ and represses p53-mediated gene expression. Cancer Biol Ther 4: 1138-1143, 2005. 